\title{
Linear Polarized Terahertz Emission under Electrical Breakdown of a Shallow Acceptor in Uniaxially Deformed Germanium
}

\author{
A.V. Andrianov*, A.O. Zakhar'in, I.N. Yassievich \\ AND N.N. ZINOV'EV
}

Ioffe Physico-Technical Institute of Russian Academy of Sciences

26 Polytehnicheskaya, St. Petersburg, 194021 Russia

We report on polarization spectra of spontaneous terahertz electroluminescence from uniaxially deformed $\mathrm{Ge}(\mathrm{Ga})$. At compressive pressure of about $3 \pm 0.3$ kbar in the [111] direction, near the impurity breakdown threshold, the linear polarization degree attains $\approx 80-90 \%$ for the main lines of the terahertz emission.

PACS numbers: 07.57.Hm, 78.60.Fi, 78.67.De

\section{Introduction}

Optical transitions between energy levels of shallow impurities in semiconductors induced by electrical breakdown of the impurities can be used as a possible operational scheme for terahertz $(\mathrm{THz})$ emitters $(0.15-10 \mathrm{THz})$. The impurity $\mathrm{THz}$ emission from Ge is quite intense and can be observed at electric fields as low as several $\mathrm{V} / \mathrm{cm}$. Although $\mathrm{THz}$ radiation accompanied by the breakdown of shallow impurities in Ge has long been found (see, e.g., [1]), its spectrum was only recently analyzed near the threshold of the breakdown of a shallow acceptor [2]. In Ref. [3], a new scheme of a $\mathrm{THz}$ laser was proposed on the basis of resonant states of a shallow acceptor in uniaxially deformed $p$-Ge. Polarization studies of the impurity $\mathrm{THz}$ emission from $p$-Ge can provide important information on the symmetry of the states involved in radiative transitions. Here we report on the measurements of polarization spectra of the spontaneous $\mathrm{THz}$ electroluminescence from uniaxially deformed $\mathrm{Ge}(\mathrm{Ga})$.

\section{Experimental details}

The studied $\mathrm{Ge}(\mathrm{Ga})$ samples had a resistivity of $20 \Omega \mathrm{cm}\left(N_{\mathrm{A}}-N_{\mathrm{D}} \approx\right.$ $\left.1.6 \times 10^{14} \mathrm{~cm}^{-3}\right)$. To create a uniaxial compressive stress in the [111] direction

*corresponding author; e-mail: alex.andrianov@mail.ioffe.ru 
the sample was embedded in an organic glass frame and the entire structure was cooled down to low temperature in a helium cryostat. The spectral measurements were performed using a Fourier spectrometer for a range of $5-350 \mathrm{~cm}^{-1}$. The measurements of the $\mathrm{THz}$ photoconductivity spectra for unstressed and stressed samples, and a comparison of the results with published data [4] were used for calibration and measurements of stress in $p$-Ge samples achieved in our setup. These results show that the level of the stress is about $3 \pm 0.3 \mathrm{kbar}$ at $T=5 \mathrm{~K}$. $\mathrm{THz}$ radiation was detected by cooled $\mathrm{Si}$ bolometer using lock-in technique. For the polarization resolved measurements we used a linear polarizer placed in front of the Fourier spectrometer. The sample holder could be rotated around optical axis to adjust the chosen polarization geometry of the emission against the deformation axis ( $\boldsymbol{e} \| P$ and $\boldsymbol{e} \perp P$, respectively).

\section{Experimental results and discussion}

$\mathrm{THz}$ emission from unstressed $\mathrm{Ge}(\mathrm{Ga})$ was unpolarized and a typical spectrum of the emission near the impurity breakdown threshold is shown in Fig. 1a. The main narrow line with the peak at $\approx 8.3 \mathrm{meV}$ corresponds to the optical transitions from the second excited acceptor state $2 \Gamma_{8}^{-}$to the ground state $1 \Gamma_{8}^{+}$ (see Fig. 1b). Hereinafter, we use commonly accepted classification of the acceptor states in the crystals of the $O_{h}$ symmetry (see, e.g., $[5,6]$ ). The high-energy asymmetry of the main line is due to the contributions of a narrow line with a peak at $\approx 9.7 \mathrm{meV}$ and a broad line with the peak at $\approx 13 \mathrm{meV}$. The line at $9.7 \mathrm{meV}$ corresponds to the transitions from one of the upper excited acceptor states (e.g. from the $4 \Gamma_{8}^{-}$state) to the ground state. The emission at $13 \mathrm{meV}$ is caused by the transitions of hot holes from the valence band to the impurity ground state (see [2] for more details). It is necessary to note that the transitions from the first excited state of the acceptor $\left(1 \Gamma_{8}^{-}\right)$do not contribute to the emission spectrum (Fig. 1) because of the oscillator strength for the $1 \Gamma_{8}^{-} \rightarrow 1 \Gamma_{8}^{+}$transition is almost 40 times lower than the value for the $2 \Gamma_{8}^{-} \rightarrow 1 \Gamma_{8}^{+}$transition [5].

Figure 2a shows the emission spectra of uniaxially deformed $\mathrm{Ge}(\mathrm{Ga})$ measured near the impurity breakdown threshold, at $E=5.2 \mathrm{~V} / \mathrm{cm}$. The main narrow line with the peak at $7.6 \mathrm{meV}$ observed at $e \perp P$ geometry is polarized across the deformation with the polarization degree of $\approx 93 \%$. The spectrum also includes a narrow line with the peak at $8.8 \mathrm{meV}$ and a broad line with the peak at $\approx 10.3 \mathrm{meV}$. The line at $10.3 \mathrm{meV}$ dominates in the spectrum measured at the $\boldsymbol{e} \| P$ geometry and its polarization degree along the deformation axis is $\approx 80 \%$.

We attribute the line with the peak at $7.6 \mathrm{meV}$ to the transitions from the $1\left(\Gamma_{5}^{-}+\Gamma_{6}^{-}\right)$acceptor excited state to the $1 \Gamma_{4}^{+}$acceptor ground state (see Fig. $2 \mathrm{~b}$ ). This interpretation is supported by the spectral position of this line that agrees with the calculations $[6]$ and by its high degree of linear polarization $(\approx 93 \%)$ across the deformation axis. We have to emphasize that the domination of this line in the emission spectrum at $e \perp P$ polarization indicates that the ratio of oscillator strengths for optical transitions from the first and second excited acceptor 

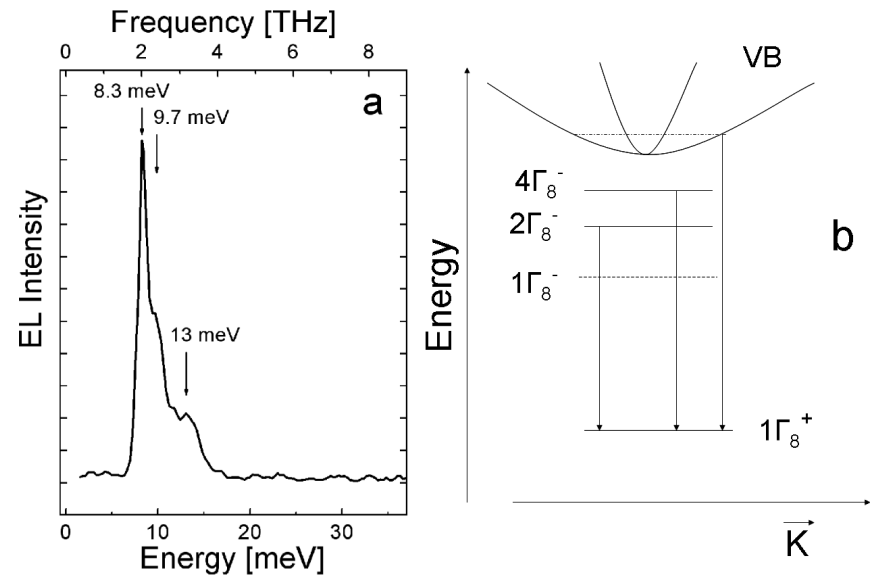

Fig. 1. (a) Terahertz electroluminescence spectrum of an unstressed $\mathrm{Ge}(\mathrm{Ga})$ sample measured with the spectral resolution of $\approx 0.6 \mathrm{meV}$ at $T=5 \mathrm{~K}$ and $E=7.5 \mathrm{~V} / \mathrm{cm}$. (b) A schematic picture of the optical transitions responsible for the $\mathrm{THz}$ emission near the threshold of the impurity breakdown in $\mathrm{Ga}(\mathrm{Ga})$.
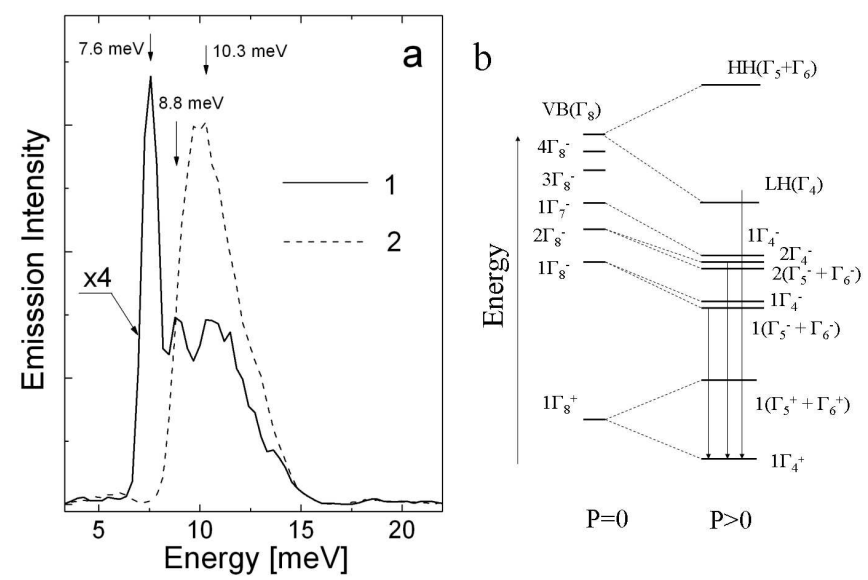

Fig. 2. (a) Spectra of $\mathrm{THz}$ electroluminescence of a $\mathrm{Ge}(\mathrm{Ga})$ sample uniaxially deformed in the [111] direction. $T=5 \mathrm{~K}, E=5.2 \mathrm{~V} / \mathrm{cm}$, the uniaxial pressure is $3 \pm 0.3 \mathrm{kbar}$ (see text), spectral resolution is $\approx 0.6 \mathrm{meV} .1-$ the $e \perp P$ polarization geometry, 2 - the e $\| P$ polarization geometry. (b) A schematic picture of ground and several excited states of a shallow acceptor in Ge under uniaxial deformation in the [111] direction. Arrows show major optical transitions, which form the $\mathrm{THz}$ electroluminescence spectrum under the impurity breakdown.

states changes substantially under the uniaxial deformation. That is the oscillator strength for the $1\left(\Gamma_{5}^{-}+\Gamma_{6}^{-}\right) \rightarrow 1 \Gamma_{4}^{+}$transition gets higher than the value for the $2\left(\Gamma_{5}^{-}+\Gamma_{6}^{-}\right) \rightarrow 1 \Gamma_{4}^{+}$transition at the stress of $\approx 3$ bar. The recent calcula- 
tions [7] support our conclusion about the strain induced increase in the oscillator strength of optical transitions from the first excited acceptor state. The line at $8.8 \mathrm{meV}$ (Fig. 2a) corresponds to the optical transitions from the $\left(\Gamma_{5}^{-}+\Gamma_{6}^{-}\right)$and $\Gamma_{4}^{-}$excited states, arising from the $2 \Gamma_{8}^{-}$and $1 \Gamma_{7}^{-}$states, to the acceptor ground state. The broad line at $10.3 \mathrm{meV}$ can be caused by the transitions of nonequilibrium holes from the $\Gamma_{4}$ subband of the valence band to the acceptor ground state. We cannot exclude also a contribution to the emission at $10.3 \mathrm{meV}$ from optical transitions between resonant acceptor states, which originate from upper excited acceptor states under uniaxial pressure exceeding $2 \mathrm{kbar}$ and concentrate near the bottom of the light hole band [4], and the acceptor ground state. Such transitions between resonant and localized acceptor states can be responsible for the strong polarization of the emission at $10.3 \mathrm{meV}$ (see Fig. 2a). Figure 3a shows the emis-
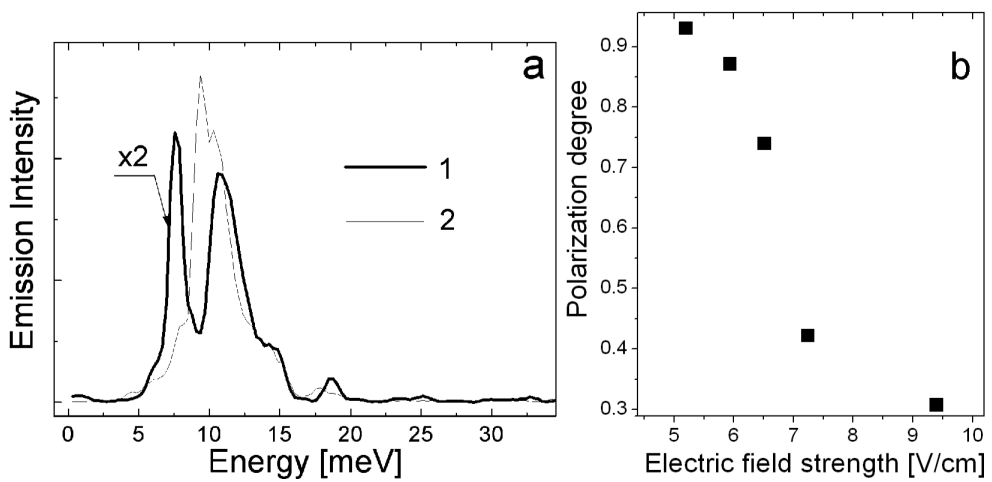

Fig. 3. (a) Terahertz electroluminescence spectra of uniaxially deformed $\mathrm{Ge}(\mathrm{Ga})$ for $E=7.3 \mathrm{~V} / \mathrm{cm}, T=5 \mathrm{~K}$ and spectral resolution of $\approx 0.6 \mathrm{meV} .1-$ the $\boldsymbol{e} \perp P$ polarization geometry, $2-$ the $e \| P$ polarization geometry. (b) Electric field dependence of the linear polarization degree of the $\mathrm{THz}$ emission at the $7.6 \mathrm{meV}$ line in uniaxially deformed $\mathrm{Ge}(\mathrm{Ga})$.

sion spectra at the electric field of $7.3 \mathrm{~V} / \mathrm{cm}$. The narrow line at $7.6 \mathrm{meV}$ remains predominantly polarized across the deformation axis, but its polarization degree decreases to $\approx 42 \%$. The decrease in the polarization degree of this line occurs due to an increase in the contribution of the $1 \Gamma_{4}^{-} \rightarrow 1 \Gamma_{4}^{+}$optical transitions from the higher excited state $1 \Gamma_{4}^{-}$, which is close in energy to the $1\left(\Gamma_{5}^{-}+\Gamma_{6}^{-}\right)$state. The $1 \Gamma_{4}^{-} \rightarrow 1 \Gamma_{4}^{+}$transition is allowed at both $\boldsymbol{e} \| P$ and $\boldsymbol{e} \perp P$ polarizations, and the relative population of the $1 \Gamma_{4}^{-}$state increases with the increase in effective electron temperature. It results in a reduction of the total polarization of the line at $7.6 \mathrm{meV}$ with the increase in the electric field as Fig. 3b demonstrates. The emission behavior of the broad line with the electric field increase is more complicated. As can be seen from Fig. 3a, a structure appears here, namely: features at $\approx 9.4 \mathrm{meV}$ and $\approx 10.3 \mathrm{meV}$, which are observed at the $e \| P$ polarization and 
have the polarization degrees of $\approx 78 \%$ and $\approx 44 \%$, respectively, at $E=7.3 \mathrm{~V} / \mathrm{cm}$. Also a feature at $\approx 10.8 \mathrm{meV}$ is seen at the $\boldsymbol{e} \perp P$ polarization. As a whole, the polarization of the $\mathrm{THz}$ emission in the region of $\approx 10 \mathrm{meV}$ decreases with the increase in the electric field strength. Most likely these regularities are also caused by influence of heating effects of nonequilibrium holes under the increase in the electric field. Possibly the feature at $9.4 \mathrm{meV}$ is related to transitions from upper excited acceptor states of $\Gamma_{4}^{-}$symmetry, whose relative population increases with the electric field increase, to the $1 \Gamma_{4}^{+}$acceptor ground state. The broad line at $10.8 \mathrm{meV}$ could be caused by transitions between the heavy and light hole subbands. Such optical transitions should be predominantly polarized across the deformation axis and, therefore, their contribution will give a decrease in the total polarization of the $\mathrm{THz}$ emission in the region of $\approx 10 \mathrm{meV}$.

\section{Conclusion}

We have observed new optical properties of $\mathrm{THz}$ emission from uniaxially deformed $\mathrm{Ge}(\mathrm{Ga})$ at the conditions of impact impurity breakdown. The main feature is a strong linear polarization of $\mathrm{THz}$ emission bands with different alignment of polarization vector against the deformation axis. The observed emission bands correspond to the optical transitions of holes between single excited states and the ground state of the acceptor and the transitions of holes from the valence band to the impurity ground state. The polarization level decays under electric field increase due to rise of electron temperature. The population of high energy states increases the contribution of optical transitions with competing polarization selection rules.

\section{Acknowledgments}

The work was supported by Russian Basic Research Fund (project No. 0502-17770).

\section{References}

[1] S.H. Koenig, R.D. Brown, Phys. Rev. Lett. 4, 170 (1960).

[2] A.V. Andrianov, A.O. Zakhar'in, I.N. Yassievich, N.N. Zinov'ev, JETP Lett. 79, $365(2004)$.

[3] M.A. Odnobludov, I.N. Yassievich, M.S. Kagan, Yu.M. Galperin, K.A. Chao, Phys. Rev. Lett. 83, 644 (1999).

[4] Ya.E. Pokrovskii, N.A. Khval'kovskii, Semiconductors 39, 182 (2005).

[5] P. Clauws, J. Broeckx, E. Rotsaert, J. Vennik, Phys. Rev. B 38, 12377 (1988).

[6] R. Buczko, Nuovo Cimento 9, 669 (1987).

[7] D.V. Kozlov, JETP Lett. 85, 247 (2007). 